\title{
Rosiglitazone may reduce non-melanoma skin cancer risk in Taiwanese
}

Chin-Hsiao Tseng ${ }^{1,2,3}$

\begin{abstract}
Background: Whether rosiglitazone may affect the risk of non-melanoma skin cancer (NMSC) has not been investigated. Methods: The reimbursement databases of all Taiwanese diabetic patients from 1996 to 2009 were retrieved from the National Health Insurance. An entry date was set at 1 January 2006 and a total of 886418 patients with type 2 diabetes were followed up for NMSC incidence until the end of 2009. Incidences for ever-users, never-users and subgroups of rosiglitazone exposure (using tertile cutoffs of duration of therapy and cumulative dose) were calculated and hazard ratios estimated by Cox regression. Additional models were created as sensitivity analyses.

Results: There were 103097 ever-users and 783321 never-users, respective numbers of incident NMSC 250 (0.24\%) and 2084 (0.27\%), and respective incidence 68.90 and 76.77 per 100000 person-years. Although the overall hazard ratio was not significant in the unadjusted, age-sex-adjusted or fully adjusted model, the risk was significantly lower in the third tertile of duration of therapy and cumulative dose, with significant $P$ for trends. The fully adjusted hazard ratio (95\% confidence interval) for a duration of therapy $>13.77$ months and a cumulative dose of $>1752 \mathrm{mg}$ was 0.723 (0.566, $0.923)$ and $0.783(0.618,0.993)$, respectively. The findings were supported by various sensitivity analyses.
\end{abstract}

Conclusions: Rosiglitazone may reduce the risk of NMSC, but further confirmation is required.

Keywords: Diabetes mellitus, Non-melanoma skin cancer, Rosiglitazone, Taiwan

\section{Background}

All three isotypes of peroxisome proliferator-activator receptors (PPARs), i.e., PPAR $\alpha$, PPAR $\beta / \delta$ and PPAR $\gamma$, are expressed in human skin keratinocytes [1,2]. They play important roles in skin barrier permeability, proliferation inhibition, differentiation promotion, immune regulation, and sebum production $[1,3]$.

$\operatorname{PPAR} \beta / \delta$ is the most predominant isotype in human keratinocytes, and it is upregulated during wound healing [1]. PPAR $\alpha$ is closely related to lipid metabolism; and plays an important role in skin barrier development [1]. PPAR $\gamma$ activation has been shown to inhibit proliferation, promote differentiation and induce apoptosis in various malignant tissues; and its agonists have been used as therapeutic agents for psoriasis, a benign skin disease characterized by epidermal hyperplasia [1,4-6].

\footnotetext{
Correspondence: ccktsh@ms6.hinet.net

'Department of Internal Medicine, National Taiwan University College of

Medicine, No. 7 Chung-Shan South Road, Taipei (100), Taiwan

2Division of Endocrinology and Metabolism, Department of Internal

Medicine, National Taiwan University Hospital, Taipei, Taiwan

Full list of author information is available at the end of the article
}

The use of PPAR agonists or antagonists in the treatment of many skin diseases including acne vulgaris, psoriasis, benign skin tumors and skin cancer is of clinical importance but still awaits in-depth investigation [2]. Whether PPAR $\gamma$ has a role in the prevention or treatment of skin cancer is under debate [2]. Reduced PPAR $\gamma$ activity is noted in mice susceptible to skin cancer induced by dimethylbenz[a]anthracene [7], but activation of PPAR $\gamma$ with rosiglitazone treatment does not prevent the development of skin tumors induced by ultraviolet light or chemical such as dimethylbenz[a]anthracene/12-O-tetradecanoylphorbol-13-acetate in mice [8]. However, a later study by the same group did show that rosiglitazone may reduce the occurrence of skin cancer in transgenic mice overexpressing insulin-like growth factor 1 (IGF1) [9].

To our knowledge, there is only one paper evaluating the risk of non-melanoma skin cancer (NMSC) in patients who had used thiazolidinediones (a class of PPAR $\gamma$ agonists used for glycemic control in patients with type 2 diabetes mellitus) by using the General Practice Research Database in the United Kingdoms [10]. The investigators 
reported a lack of association without specifying the various thiazolidinediones used by the patients.

In an early study conducted in keratinocytes in monolayer culture and in human whole skin organ culture, rosiglitazone (one of the thiazolidinediones used in clinical practice) inhibits proliferation, motility, and matrix metalloproteinase production in keratinocytes more effectively than the other PPARy agonist pioglitazone does [11]. In consideration of the better performance of rosiglitazone on the inhibition of keratinocyte proliferation [11], and the recently reported potential risk of bladder cancer related to pioglitazone [12-14], the present study aimed at evaluating the association between rosiglitazone use and NMSC in Taiwanese patients with type 2 diabetes mellitus by using the reimbursement databases of the National Health Insurance (NHI).

\section{Methods}

The study was approved by an ethic review board of the National Health Research Institutes with registered approval number 99274 .

Since March 1995, a compulsory and universal system of health insurance (the so-called NHI) was implemented in Taiwan. All contracted medical institutes must submit computerized and standard claim documents for reimbursement. More than $99 \%$ of citizens are enrolled in the NHI, and $>98 \%$ of the hospitals nationwide are under contract with the NHI. The average number of annual physician visits in Taiwan is one of the highest around the world, at approximately 15 visits per year per capita in 2009 .

The National Health Research Institutes is the only organization approved, as per local regulations, for handling the NHI reimbursement databases for academic research. The databases contain detailed records on every visit for each patient, including outpatient visits, emergency department visits and hospital admission. The databases also include principal and secondary diagnostic codes, prescription orders, and claimed expenses.

The identification information of the individuals was scrambled for the protection of privacy. Diabetes was coded 250.XX and NMSC 173, based on the International Classification of Diseases, Ninth Revision, Clinical Modification (ICD-9-CM).

The databases of all patients who had been diagnosed with diabetes and under treatment with either oral antidiabetic agents or insulin during the period of 1996-2009 from the whole nation, and who remained enrolled in the NHI after $2006(n=1446414)$ were first retrieved. A total of 235287 patients who had ever been treated with pioglitazone were then excluded to avoid the possible contamination by its use because it may increase the risk of some cancer like bladder cancer [15]. The selected entry date was 1 January 2006. After excluding patients who had a diagnosis of diabetes after the year $2006(n=342351)$, patients who held a Severe Morbidity Card as having type 1 diabetes $(n=7120$, in Taiwan, patients with type 1 diabetes were issued a so-called "Severe Morbidity Card" after certified diagnosis and they were waived for much of the co-payments), patients having a diagnosis of NMSC before $2006(n=6297)$, those who died $(n=96320)$ or withdrew from the NHI $(n=12502)$ before entry date, duplicated identification number $(n=106)$, and unclear information on date of birth or sex $(n=5123)$, a total of 886418 patients with a diagnosis of type 2 diabetes mellitus and under therapy with oral anti-diabetic agents (except pioglitazone) or insulin were recruited.

Those who had ever been prescribed with rosiglitazone before entry date were defined as ever-users; and neverusers were defined as those who had never been prescribed with rosiglitazone before entry date. To evaluate whether a dose-response relationship could be seen between rosiglitazone and NMSC, the tertile cutoffs for duration of therapy in months and cumulative dose in $\mathrm{mg}$ were calculated from the databases and used for analyses.

An entry date at the beginning of 2006 was used based on the following reasons: 1) Because rosiglitazone was marketed in 2001 in Taiwan, this entry date, being in the middle of the marketing date of rosiglitazone in Taiwan and the ending date of the available NHI databases in 2009, provided a longest exposure of 4 to 5 years at entry and at the same time a longest follow-up duration of 4 years; and 2) The issue of bladder cancer associated with pioglitazone noted in the PROspective pioglitAzone Clinical Trial In macroVascular Events (PROactive) was published in 2005 [16], and in 2007, the safety of rosiglitazone has been challenged with a risk of acute myocardial infarction [17]. These had caused tremendous prescription behavior changes in the physicians to withdraw thiazolidinediones including rosiglitazone and pioglitazone (troglitazone has not been marketed in Taiwan) and the patients might have stopped taking the drugs even if they were prescribed after the year 2006. Therefore, the use of a later entry date would make the estimation of the duration of therapy and cumulative dose of rosiglitazone less reliable. In addition, this would also shorten the follow-up duration for cancer incidence.

All comorbidities and covariates were determined as a status/diagnosis before the entry date. The ICD-9-CM codes for the comorbidities were [18-21]: nephropathy 580-589, hypertension 401-405, chronic obstructive pulmonary disease (a surrogate for smoking) 490-496, cerebrovascular disease 430-438, ischemic heart disease 410-414, peripheral arterial disease 250.7, 785.4, 443.81 and 440-448, eye disease $250.5,362.0,369,366.41$ and 365.44, obesity 278, dyslipidemia 272.0-272.4, and cancer other than NMSC 140-208 (excluding 173). Medications included sulfonylurea, metformin, insulin, and acarbose. 
Baseline characteristics between ever-users and neverusers of rosiglitazone were compared by Chi-square test.

The incidence density of NMSC was calculated for ever-users and never-users and for different subgroups of exposure. Chi-square test was used to compare the distribution of incident cases of NMSC in ever-users versus never-users and among the different subgroups of dose-response parameters. The numerator for the incidence was the number of patients with incident NMSC during the 4-year follow-up, and the denominator was the person-years of follow-up. For ever-users, the follow-up duration was either censored at the date of NMSC diagnosis or at the date of the last record of the available reimbursement databases in individuals without incident NMSC. For never-users, the followup was censored at the date of rosiglitazone initiation or NMSC diagnosis or the last reimbursement record, depending on whichever occurring first. This ensured no exposure to rosiglitazone throughout the whole follow-up period until censor in the referent group of never-users.

Cox proportional hazards regression was performed to estimate the hazard ratios for NMSC for ever-users versus never-users, and for the various subgroups of doseresponse parameters. The following three models were created: 1) unadjusted; 2) adjusted for age and sex; and 3) adjusted for all variables compared previously as baseline characteristics between ever-users and never-users.

The following fully adjusted models were also created as sensitivity analyses: 1) resetting the entry date to 1 January 2005; 2) deleting patients who developed NMSC within 3 months of follow-up; 3) excluding patients with a history of any cancer before 2006; 4) including pioglitazone users in the analyses; 5) using a time-dependent approach; and 6) excluding never-users of rosiglitazone and conducting the analyses only among rosiglitazone ever-users by comparing the second and third tertiles of exposure versus the first tertiles as referents.

Analyses were conducted using SAS statistical software, version 9.3 (SAS Institute, Cary, NC). $P<0.05$ was considered statistically significant.

\section{Results}

Table 1 compares the baseline characteristics between ever-users $(n=103097)$ and never-users $(n=783321)$ of rosiglitazone. All of the variables differed significantly between the two groups. Ever-users are characterized by older age distribution, higher proportion with a diabetes duration $\geq 5$ years, higher proportions of all comorbidities and other cancer, higher proportions of using other medications.

Table 2 shows the incidences of NMSC between everusers and never-users of rosiglitazone, and among the different categories of the dose-response parameters for rosiglitazone exposure. The incidence rate in never-users and ever-users of rosiglitazone was 76.77 and 68.90 per 100000 person-years, respectively.

Table 3 shows the hazard ratios with regards to different categories of rosiglitazone exposure. In the models evaluating the overall hazard ratios for ever-users versus neverusers, the hazard ratios were not significant in all three models. However, in the models evaluating the dose-response exposure to rosiglitazone with regards to duration of therapy and cumulative dose, a significantly lower risk could be seen in the third tertiles and the trend analyses.

The sensitivity analyses are shown in Table 4. Generally speaking, the results were consistent with the full models in the original analyses (Table 3). Although the overall hazard ratios were not significant, the third tertiles of rosiglitazone exposure were generally associated with a significantly lower risk of NMSC. In the time-dependent analyses, an approximately 30\% higher risk was observed for the first tertiles of duration of therapy and cumulative dose, but the third tertiles remained significantly associated with a lower risk. In the analyses comparing the second and third tertiles of rosiglitazone exposure versus the first tertiles as referent by including only rosiglitazone ever-users, the hazard ratio ( $95 \%$ confidence interval) for the second and third tertile of duration of therapy was $0.850(0.634,1.140)(\mathrm{P}=0.2777)$ and $0.639(0.465,0.876)$ $(\mathrm{P}=0.0054)$, respectively ( $\mathrm{P}$-trend $=0.0054) ;$ and was $0.852(0.633,1.148)$ and $0.712(0.521,0.973)$, respectively, for cumulative dose (P-trend $=0.0327$ ).

\section{Discussion}

This is the first observational cohort study evaluating the risk of NMSC associated with the specific use of rosiglitazone in patients with type 2 diabetes mellitus. The findings suggested a reduced risk in patients who had used the drug for more than 13 months or with a cumulative dose of greater than $1750 \mathrm{mg}$ (Table 3). The results of such a promising effect provided rationale for further in-depth investigation on this drug in the prevention of NMSC.

The findings were consistent in various sensitivity analyses, except in the time-dependent models that showed a significantly higher risk in the first tertiles of rosiglitazone exposure (Table 4). In the analyses for the incidence of NMSC, patients in the first tertiles of rosiglitazone exposure also showed a slightly higher incidence than the referent groups of never-users of rosiglitazone (Table 2). This could result from residual confounding among users of a short duration (i.e., in the first tertiles of exposure) because rosiglitazone is always used as a second or third line drug for glucose lowering in clinical practice. At the inception of its use, patients are usually characterized by older age, a longer duration of diabetes (Table 1) and poorer glycemic control (data not available), which might 
Table 1 Baseline characteristics between never-users and ever-users of rosiglitazone

\begin{tabular}{|c|c|c|c|c|c|}
\hline \multirow[t]{2}{*}{ Variables } & \multicolumn{2}{|c|}{ Never-users } & \multicolumn{2}{|c|}{ Ever-users } & \multirow[t]{2}{*}{$P$} \\
\hline & $n$ & $\%$ & $n$ & $\%$ & \\
\hline$n=886418$ & 783321 & & 103097 & & \\
\hline \multicolumn{6}{|l|}{ Age (years) } \\
\hline$<40$ & 33874 & 4.32 & 3269 & 3.17 & $<0.0001$ \\
\hline $40-49$ & 102887 & 13.13 & 11623 & 11.27 & \\
\hline $50-59$ & 199819 & 25.51 & 27204 & 26.39 & \\
\hline $60-69$ & 200241 & 25.56 & 29608 & 28.72 & \\
\hline$\geq 70$ & 246500 & 31.47 & 31393 & 30.45 & \\
\hline Sex (men) & 399333 & 50.98 & 49282 & 47.80 & $<0.0001$ \\
\hline \multicolumn{6}{|l|}{ Diabetes duration (years) } \\
\hline$<1$ & 70381 & 8.98 & 1434 & 1.39 & $<0.0001$ \\
\hline $1-3$ & 133250 & 17.01 & 7184 & 6.97 & \\
\hline $3-5$ & 123610 & 15.78 & 11094 & 10.76 & \\
\hline$\geq 5$ & 456080 & 58.22 & 83385 & 80.88 & \\
\hline Hypertension & 443728 & 56.65 & 77740 & 75.40 & $<0.0001$ \\
\hline Chronic obstructive pulmonary disease & 114018 & 14.56 & 24177 & 23.45 & $<0.0001$ \\
\hline Cerebrovascular disease & 116947 & 14.93 & 25453 & 24.69 & $<0.0001$ \\
\hline Nephropathy & 93744 & 11.97 & 23717 & 23.00 & $<0.0001$ \\
\hline Ischemic heart disease & 174225 & 22.24 & 40511 & 39.29 & $<0.0001$ \\
\hline Peripheral arterial disease & 89324 & 11.40 & 24510 & 23.77 & $<0.0001$ \\
\hline Eye disease & 63803 & 8.15 & 21179 & 20.54 & $<0.0001$ \\
\hline Obesity & 11423 & 1.46 & 1961 & 1.90 & $<0.0001$ \\
\hline Dyslipidemia & 371714 & 47.45 & 69015 & 66.94 & $<0.0001$ \\
\hline Other cancer prior to baseline & 93619 & 11.95 & 13058 & 12.67 & $<0.0001$ \\
\hline Sulfonylurea & 577003 & 73.66 & 99140 & 96.16 & $<0.0001$ \\
\hline Metformin & 512063 & 65.37 & 96850 & 93.94 & $<0.0001$ \\
\hline Acarbose & 80640 & 10.29 & 38971 & 37.80 & $<0.0001$ \\
\hline Insulin & 102359 & 13.07 & 39119 & 37.94 & $<0.0001$ \\
\hline
\end{tabular}

themselves be associated with an increased risk of cancer development.

Exposure to ultraviolet sunlight may be the most important risk factor for skin cancer; and other risk factors may include family history, fair skin, light eyes, and immunosuppression [22]. It should be admitted that most of these potential confounders could not be adjusted for in the present study because of the lack of such information in the reimbursement databases. Therefore, the beneficial effect of rosiglitazone on the prevention of NMSC should better be confirmed in future studies considering the adjustment for these potential confounders.

Misclassification of NMSC might occur in the present study. However, such a probability was low because labeled diagnoses should be printed out in all prescriptions handed to the patients, and mislabeling of a cancer diagnosis would not be acceptable to the patients when they saw the diagnosis.
Because the present study recruited only patients with type 2 diabetes mellitus, the results should not be readily generalized to non-diabetic individuals. The animal studies conducted by $\mathrm{He}$ et al. have provided good reasons for this cautiousness. In ordinary mice treated with carcinogens, rosiglitazone does not reduce skin cancer risk [8], but rosiglitazone does reduce skin cancer risk in transgenic mice overexpressing IGF1 [9]. The authors explained that ultraviolet light and carcinogenic chemicals may activate some carcinogenic pathways enabling keratinocytes to bypass the IGF1 signaling; and that rosiglitazone may possibly inhibit the development of skin cancer through activation of AMP-activated protein kinase with subsequent inhibition of IGF1-induced mammalian target of rapamycin activity and phosphorylation of p70S6 kinase [9]. Patients with type 2 diabetes mellitus are at an increased risk of cancer and they are characterized by insulin resistance with hyperinsulinemia and hyperactivity in 
Table 2 Incidence of non-melanoma skin cancer by rosiglitazone exposure at entry

\begin{tabular}{|c|c|c|c|c|c|}
\hline Rosiglitazone use & Case number & $\begin{array}{l}\text { Incident skin } \\
\text { cancer }\end{array}$ & $\%$ & Person-years & $\begin{array}{l}\text { Incidence rate } \\
\text { (per } 100,000 \text { person-years) }\end{array}$ \\
\hline Never users & 783321 & 2084 & 0.27 & 2714745.08 & 76.77 \\
\hline Ever users & 103097 & 250 & 0.24 & 362846.42 & 68.90 \\
\hline$P$ value & & & 0.1653 & & \\
\hline \multicolumn{6}{|c|}{ Duration of therapy (months) } \\
\hline Never users & 783321 & 2084 & 0.27 & 2714745.08 & 76.77 \\
\hline$<3.73$ & 33039 & 93 & 0.28 & 112752.67 & 82.48 \\
\hline $3.73-13.77$ & 35108 & 88 & 0.25 & 123278.50 & 71.38 \\
\hline$>13.77$ & 34950 & 69 & 0.20 & 126815.25 & 54.41 \\
\hline$P$ value & & & 0.0847 & & \\
\hline \multicolumn{6}{|c|}{ Cumulative dose (mg) } \\
\hline Never users & 783321 & 2084 & 0.27 & 2714745.08 & 76.77 \\
\hline$<448$ & 32942 & 90 & 0.27 & 112711.50 & 79.85 \\
\hline $448-1752$ & 35078 & 86 & 0.25 & 123002.83 & 69.92 \\
\hline$>1752$ & 35077 & 74 & 0.21 & 127132.08 & 58.21 \\
\hline$P$ value & & & 0.2172 & & \\
\hline
\end{tabular}

IGF1 signaling [23]. Therefore, more studies are required to clarify the usefulness of rosiglitazone on the prevention of skin cancer in individuals without diabetes mellitus.

This study has several strengths. Because the databases were derived from the whole population and they spanned the whole period from the beginning of the marketing of rosiglitazone from 2001 in Taiwan to the end of 2009, there was no concern of potential selection bias related to sampling error. The databases included all claim records on outpatient visits, emergency department visits and hospital admission, and we caught the diagnoses from all sources. Cancer is considered a severe morbidity by the NHI and most medical co-payments can be waived. Furthermore, there is a low drug costsharing required by the NHI and patients with certain conditions such as low-income household, veterans or patients with prescription refills for chronic disease are exempted from the drug cost-sharing. Therefore the detection rate of NMSC would not tend to differ among different social classes. The use of medical records also reduced the potential bias related to self-reporting.

The study limitations included a lack of actual measurement data for potential confounders such as sunlight exposure, occupation history, family history, lifestyle, diet,

Table 3 Rosiglitazone exposure at entry and hazard ratios for non-melanoma skin cancer

\begin{tabular}{|c|c|c|c|c|c|c|c|c|c|}
\hline \multirow{2}{*}{$\begin{array}{l}\text { Rosiglitazone } \\
\text { use }\end{array}$} & \multicolumn{3}{|c|}{ Unadjusted } & \multicolumn{3}{|c|}{ Age-sex-adjusted } & \multicolumn{3}{|c|}{ Fully adjusted* } \\
\hline & $\mathrm{HR}$ & $95 \% \mathrm{Cl}$ & $P$ & $\mathrm{HR}$ & $95 \% \mathrm{Cl}$ & $P$ & $\mathrm{HR}$ & $95 \% \mathrm{Cl}$ & $P$ \\
\hline Ever users & 0.882 & $(0.773,1.005)$ & 0.0602 & 0.908 & $(0.796,1.036)$ & 0.1513 & 0.927 & $(0.807,1.066)$ & 0.2865 \\
\hline \multicolumn{10}{|c|}{ Duration of therapy (months) } \\
\hline$<3.73$ & 1.059 & $(0.861,1.304)$ & 0.5862 & 1.109 & $(0.901,1.365)$ & 0.3295 & 1.119 & $(0.905,1.383)$ & 0.2985 \\
\hline $3.73-13.77$ & 0.903 & $(0.730,1.118)$ & 0.3507 & 0.941 & $(0.760,1.165)$ & 0.5744 & 0.961 & $(0.773,1.196)$ & 0.7243 \\
\hline$>13.77$ & 0.703 & $(0.553,0.893)$ & 0.0039 & 0.705 & $(0.555,0.896)$ & 0.0043 & 0.723 & $(0.566,0.923)$ & 0.0094 \\
\hline P-trend & & & 0.0072 & & & 0.0171 & & & 0.0386 \\
\hline \multicolumn{10}{|c|}{ Cumulative dose (mg) } \\
\hline$<448$ & 1.025 & $(0.830,1.265)$ & 0.8221 & 1.070 & $(0.867,1.322)$ & 0.5276 & 1.081 & $(0.872,1.341)$ & 0.4773 \\
\hline $448-1752$ & 0.885 & $(0.713,1.098)$ & 0.2675 & 0.912 & $(0.735,1.132)$ & 0.4033 & 0.931 & $(0.747,1.161)$ & 0.5270 \\
\hline$>1752$ & 0.752 & $(0.596,0.948)$ & 0.0157 & 0.764 & $(0.606,0.963)$ & 0.0228 & 0.783 & $(0.618,0.993)$ & 0.0436 \\
\hline P-trend & & & 0.0139 & & & 0.0337 & & & 0.0743 \\
\hline
\end{tabular}

Referent group: never-users of rosiglitazone; HR: hazard ratio, Cl: confidence intervals.

*Adjusted for all variables in Table 1. 


\section{Table 4 Sensitivity analyses estimating hazard ratios for non-melanoma skin cancer associated with rosiglitazone exposure}

\begin{tabular}{|c|c|c|c|c|c|c|c|c|c|c|c|c|c|c|c|}
\hline \multirow[t]{2}{*}{ Rosiglitazone use } & \multicolumn{3}{|c|}{$\begin{array}{l}\text { Setting entry date to } 1 \\
\text { January } 2005\end{array}$} & \multicolumn{3}{|c|}{$\begin{array}{l}\text { Deleting patients who } \\
\text { developed skin cancer within } \\
3 \text { months of follow-up }\end{array}$} & \multicolumn{3}{|c|}{$\begin{array}{l}\text { Excluding patients with a history } \\
\text { of any cancer before } 2006\end{array}$} & \multicolumn{3}{|c|}{$\begin{array}{l}\text { Including pioglitazone } \\
\text { users in the analyses }\end{array}$} & \multicolumn{3}{|c|}{ Time-dependent models } \\
\hline & $\mathrm{HR}$ & $95 \% \mathrm{Cl}$ & $P$ & $\mathrm{HR}$ & $95 \% \mathrm{Cl}$ & $P$ & $\mathrm{HR}$ & $95 \% \mathrm{Cl}$ & $P$ & $\mathrm{HR}$ & $95 \% \mathrm{Cl}$ & $P$ & HR & $95 \% \mathrm{Cl}$ & $P$ \\
\hline Ever users & 0.986 & $(0.882,1.102)$ & 0.7977 & 0.933 & $(0.809,1.075)$ & 0.3368 & 0.951 & $(0.834,1.083)$ & 0.4469 & 0.938 & $(0.835,1.055)$ & 0.2863 & 0.909 & $(0.785,1.051)$ & 0.1981 \\
\hline \multicolumn{16}{|c|}{ Duration of therapy (months) } \\
\hline$<3.73$ & 1.177 & $(0.975,1.421)$ & 0.0903 & 1.119 & $(0.900,1.392)$ & 0.3106 & 1.055 & $(0.855,1.302)$ & 0.6199 & 1.114 & $(0.930,1.336)$ & 0.2411 & 1.287 & $(1.050,1.579)$ & 0.0153 \\
\hline $3.73-13.77$ & 1.112 & $(0.939,1.318)$ & 0.2189 & 0.989 & $(0.792,1.234)$ & 0.9194 & 1.096 & $(0.906,1.325)$ & 0.3452 & 1.025 & $(0.862,1.219)$ & 0.7781 & 1.059 & $(0.851,1.317)$ & 0.6099 \\
\hline$>13.77$ & 0.787 & $(0.662,0.935)$ & 0.0065 & 0.713 & $(0.554,0.918)$ & 0.0087 & 0.737 & $(0.592,0.916)$ & 0.0060 & 0.718 & $(0.589,0.874)$ & 0.0010 & 0.583 & $(0.457,0.740)$ & $<0.0001$ \\
\hline P-trend & & & 0.1215 & & & 0.0484 & & & 0.0871 & & & 0.0186 & & & 0.0024 \\
\hline \multicolumn{16}{|c|}{ Cumulative dose $(\mathrm{mg})$} \\
\hline$<448$ & 1.138 & $(0.939,1.379)$ & 0.1883 & 1.092 & $(0.876,1.361)$ & 0.4336 & 0.985 & $(0.793,1.223)$ & 0.8882 & 1.059 & $(0.880,1.275)$ & 0.5418 & 1.286 & $(1.030,1.607)$ & 0.0264 \\
\hline $448-1752$ & 1.099 & $(0.929,1.301)$ & 0.2722 & 0.923 & $(0.735,1.159)$ & 0.4892 & 1.105 & $(0.915,1.334)$ & 0.3015 & 1.011 & $(0.849,1.203)$ & 0.9047 & 0.983 & $(0.775,1.247)$ & 0.8876 \\
\hline$>1752$ & 0.815 & $(0.687,0.966)$ & 0.0186 & 0.798 & $(0.627,1.016)$ & 0.0672 & 0.780 & $(0.630,0.965)$ & 0.0224 & 0.773 & $(0.638,0.936)$ & 0.0084 & 0.614 & $(0.476,0.792)$ & 0.0002 \\
\hline P-trend & & & 0.1839 & & & 0.0971 & & & 0.1685 & & & 0.0482 & & & 0.0041 \\
\hline
\end{tabular}

Referent group: never-users of rosiglitazone; HR: hazard ratio, Cl: confidence intervals.

In models excluding patients not treated with rosiglitazone and using the first tertile of exposure as referent, the hazard ratio ( $95 \%$ confidence interval) for the second and third tertile of duration of therapy was 0.850 $(0.634,1.140)(P=0.2777)$ and $0.639(0.465,0.876)(P=0.0054)$, respectively $(P$-trend $=0.0054)$; and was $0.852(0.633,1.148)$ and $0.712(0.521,0.973)$, respectively, for cumulative dose $(P$-trend $=0.0327)$. 
and genetic parameters. In addition, we did not have biochemical data for evaluating their impact. Another limitation is the lack of information on the histopathology, grading and staging of NMSC.

In summary, this study first reports a potential beneficial effect of the use of rosiglitazone on the prevention of NMSC in patients who have been using the medication for more than 13 months or with a cumulative dose of greater than $1750 \mathrm{mg}$.

\section{Conclusions}

This large population-based retrospective cohort study in Taiwan suggests that prolonged use of rosiglitazone in patients with type 2 diabetes mellitus may be associated with a lower risk of NMSC. Exploring the potential use of rosiglitazone as a preventive and/or therapeutic agent for NMSC is an interesting issue with clinical importance. However, more researches are required to confirm the findings of the present study.

\section{Abbreviations}

ICD-9-CM: International Classification of Diseases, Ninth Revision, Clinical Modification; IGF1: Insulin-like growth factor 1; NHI: National Health Insurance; NMSC: Non-melanoma skin cancer; PPAR: Peroxisome proliferator-activator receptors.

\section{Competing interests}

The author declares that he has no competing interests.

\section{Author's contributions}

$\mathrm{CH}$ researched data and wrote manuscript.

\section{Acknowledgments}

The study was supported by the National Science Council (NSC102-2314-B002-067) and the Ministry of Science and Technology (MOST 103-2314-B002-187-MY3) of Taiwan. It is based in part on data from the National Health Insurance Research Database provided by the Bureau of National Health Insurance, Department of Health and managed by National Health Research Institutes (Registered number 99274). The interpretation and conclusions contained herein do not represent those of Bureau of National Health Insurance, Department of Health or National Health Research Institutes. The author thanks Professor Tien-Shang Huang for his generous comments to the contents of the paper and Miss Ting-Ting Chang for her excellent help in the management of the reimbursement databases. The publication fee of this paper was supported by the Weng Yuan Endocrine Fund.

\section{Author details \\ 'Department of Internal Medicine, National Taiwan University College of Medicine, No. 7 Chung-Shan South Road, Taipei (100), Taiwan. ${ }^{2}$ Division of Endocrinology and Metabolism, Department of Internal Medicine, National Taiwan University Hospital, Taipei, Taiwan. ${ }^{3}$ Division of Environmental Health and Occupational Medicine of the National Health Research Institutes, Taipei, Taiwan.}

Received: 11 October 2013 Accepted: 29 January 2015

Published online: 06 February 2015

\section{References}

1. Kuenzli S, Saurat JH. Peroxisome proliferator-activated receptors in cutaneous biology. Br J Dermatol. 2003;149:229-36.

2. Michalik $L$, Wahli W. Peroxisome proliferator-activated receptors (PPARs) in skin health, repair and disease. Biochim Biophys Acta. 2007;1771:991-8.

3. Trivedi NR, Cong Z, Nelson AM, Albert AJ, Rosamilia LL, Sivarajah S, et al. Peroxisome proliferator-activated receptors increase human sebum production. J Invest Dermatol. 2006;126:2002-9.
4. Bhagavathula N, Nerusu KC, Reddy M, Ellis CN, Chittiboyina A, Avery M, et al. BP-1 107 [\{2-[4-(2,4-dioxo-thiazolidin-5-ylmethyl)-phenoxy]-ethyl\}-methylamide]: a novel synthetic thiazolidinedione that inhibits epidermal hyperplasia in psoriatic skin-severe-combined immunodeficient mouse transplants after topical application. J Pharmacol Exp Ther. 2005;315:996-1004.

5. Malhotra S, Bansal D, Shafig N, Pandhi P, Kumar B. Potential therapeutic role of peroxisome proliferator activated receptor-gamma agonists in psoriasis. Expert Opin Pharmacother. 2005;6:1455-61.

6. Varani J, Bhagavathula N, Ellis CN, Pershadsingh HA. Thiazolidinediones: potential as therapeutics for psoriasis and perhaps other hyperproliferative skin disease. Expert Opin Investig Drugs. 2006;15:1453-68.

7. Nicol CJ, Yoon M, Ward JM, Yamashita M, Fukamachi K, Peters JM, et al. PPARgamma influences susceptibility to DMBA-induced mammary, ovarian and skin carcinogenesis. Carcinogenesis. 2004;25:1747-55.

8. He G, Muga S, Thuillier P, Lubet RA, Fischer SM. The effect of PPARgamma ligands on UV- or chemically-induced carcinogenesis in mouse skin. Mol Carcinog. 2005;43:198-206.

9. He G, Sung YM, Digiovanni J, Fischer SM. Thiazolidinediones inhibit insulin-like growth factor-i-induced activation of p70S6 kinase and suppress insulin-like growth factor-l tumor-promoting activity. Cancer Res. 2006;66:1873-8.

10. van Staa TP, Patel D, Gallagher AM, de Bruin ML. Glucose-lowering agents and the patterns of risk for cancer: a study with the General Practice Research Database and secondary care data. Diabetologia. 2012;55:654-65.

11. Bhagavathula N, Nerusu KC, Lal A, Ellis CN, Chittiboyina A, Avery MA, et al. Rosiglitazone inhibits proliferation, motility, and matrix metalloproteinase production in keratinocytes. J Invest Dermatol. 2004;122:130-9.

12. Tseng $\mathrm{CH}$. Pioglitazone and bladder cancer: a population-based study of Taiwanese. Diabetes Care. 2012;35:278-80.

13. Tseng $\mathrm{CH}$. A review on thiazolidinediones and bladder cancer in human studies. J Environ Sci Health C Environ Carcinog Ecotoxicol Rev. 2014;32:1-45.

14. Tseng $\mathrm{CH}$, Tseng FH. Peroxisome proliferator-activated receptor agonists and bladder cancer: lessons from animal studies. J Environ Sci Health C Environ Carcinog Ecotoxicol Rev. 2012;30:368-402.

15. Lewis JD, Ferrara A, Peng T, Hedderson M, Bilker WB, Quesenberry Jr CP, et al. Risk of bladder cancer among diabetic patients treated with pioglitazone: interim report of a longitudinal cohort study. Diabetes Care. 2011;34:916-22

16. Dormandy JA, Charbonnel B, Eckland DJ, Erdmann E, Massi-Benedetti M, Moules IK, et al. Secondary prevention of macrovascular events in patients with type 2 diabetes in the PROactive Study (PROspective pioglitAzone Clinical Trial In macroVascular Events): a randomised controlled trial. Lancet. 2005;366:1279-89.

17. Nissen SE, Wolski K. Effect of rosiglitazone on the risk of myocardial infarction and death from cardiovascular causes. N Engl J Med. 2007;356:2457-71.

18. Tseng CH. Benign prostatic hyperplasia is a significant risk factor for bladder cancer in diabetic patients: a population-based cohort study using the National Health Insurance in Taiwan. BMC Cancer. 2013;13:7.

19. Tseng $\mathrm{CH}$. Thyroid cancer risk is not increased in diabetic patients. PLoS One. 2012;7:e53096.

20. Tseng CH. New-onset diabetes with a history of dyslipidemia predicts pancreatic cancer. Pancreas. 2013;42:42-8.

21. Tseng CH. Diabetes, insulin use, and non-Hodgkin lymphoma mortality in Taiwan. Metabolism. 2012;61:1003-9.

22. Pelucchi C, Di Landro A, Naldi L, La Vecchia C, Oncology Study Group of the Italian Group for Epidemiologic Research in Dermatology (GISED). Risk factors for histological types and anatomic sites of cutaneous basal-cell carcinoma: an italian case-control study. J Invest Dermatol. 2007;127:935-44.

23. Arcidiacono B, liritano S, Nocera A, Possidente K, Nevolo MT, Ventura V, et al. Insulin resistance and cancer risk: an overview of the pathogenetic mechanisms. Exp Diabetes Res. 2012;2012:789174. 